\title{
Comparing the Effect of Acupressure and Cryotherapy on the Pain Caused by Removal of Chest Drain Tube in Crossiark the Elderly Patients Undergoing Open Heart Surgery
}

\author{
Farideh Bastani $^{1}$, Sakineh Hajizadeh ${ }^{1^{*}}$, Kiarash Sa`atchi ${ }^{2}$, Hamid Haghani ${ }^{3}$ \\ 1. Department of Geriatric Nursing, School of Nursing \& Midwifery, Iran University of Medical Sciences, Tehran, Iran \\ 2. Faculty of Traditional Medicine, Iran University of Medical Sciences, Tehran, Iran \\ 3. Department of Biostatistics, Faculty of Health, Iran University of Medical Sciences, Tehran, Iran.
}

ditation: Bastani, F, Hajizadeh, S, Sa atchi, K \& Haghani, H 2016, 'Comparing the effect of acupressure and cryotherapy on the pain caused by removal of chest drain tube in the elderly patients undergoing open heart surgery', Journal of Client-Centered Nursing Care, vol. 2, no. 1, pp. 37-52. https://doi.org/10.32598/jccnc.2.1.37

: https://doi.org/10.32598/jccnc.2.1.37

Article info:

Received: 13 Jun. 2015

Accepted: 05 Sep. 2015

Keywords:

Ageing, Chest tube, Pain, Acupressure, Cold therapy (Cryotherapy)

\begin{abstract}
A B S T RA C T
Background: Aging has a growing trend in the world. The elderly experience a high incidence of hospitalization and coronary bypass surgery compared with other age groups, and pain is the most common complaint in these patients. This research was conducted to compare the effect of acupressure and cryotherapy on the pain of removing drain tube from the chest of the elderly who have undergone open heart surgery.

Methods: In this quasi-experimental clinincal trial, 150 elderly people undergoing coronary artery bypass grafting with drain tube in their chests and hospitalized in the Intensive Care Unit of Shahid Rajaei Hospital (affiliated to Alborz University of Medical Sciences and Health and Treatment Services, Tehran, Iran) were selected through random sampling method. Then, they were divided into 3 groups of acupressure, cryotherapy, and control. The acupressure group received pressure on ST36, P6, LI4 points. The cryotherapy group received cold therapy through applying three packs of cooling gel, and the control group received routine postoperative cares. The pain intensity was measured 20 minutes before the insertion of the tube, during insertion, and 10 minutes after removal of the drain tube. The tools for collecting data were abbreviated mental test form, demographic questionnaire, and visual analoge scale. The obtained data were analyzed through descriptive and inferential statistics by SPSS version 20 .
\end{abstract}

Results: In terms of demographic characteristics, the patients were homogenous. Before the intervention, there were no significant differences among three groups in terms of pain intensity $(\mathrm{P}=0.93)$. However, after the intervention, pain intensity, immediately after, and 10 minutes after removal of chest tube, significantly reduced in the experimental groups compared with the control group $(\mathrm{P}<0.001)$. In general, reduction of pain intensity was higher in the acupressure group compared with the other two groups $(\mathrm{P}<0.001)$.

Conclusion: Applying acupressure on ST36, LI4, and P6 points is effective in reducing the intensity of pain caused by removal of drain tube from the chest of the elderly patients. It is recommended as a safe and inexpensive drug-free approach to control pain caused by the removal of drain tube.

\section{* Corresponding Author:}




\section{Background}

geing has a growing trend in the world 1 (Rouch 2014). According to a report prepared in 2012 by WHO, it was estimated that the world elderly population who are 60 years of age and older increases from $11 \%$ in 2000 to $22 \%$ by 2050 and the number of people above 80 years of age increases by approximately 4 times from 2000 to 2050 , i.e. reaches up to 395 million. The elderly experience a higher incidence of hospitalization, operations, and medical advice compared to other age groups (Eliopoulos 2014). Although the elderly account for about $50 \%$ of patients in the intensive care units (ICUs), limited research has been conducted on them (Foreman et al. 2010). Today, coronary artery bypass graft (CABG) is a conventional intervention treatment carried out on patients with coronary artery disease. Although many advances have made in drug treatment and catheterization procedures, surgery interventions are still considered the basic treatment for these diseases (Hosseinian et al. 2014).

One of the operations that require thoracic cage surgery and sternotomy is open heart surgery, which has increased significantly in recent years (Moemenabadi 2010). This operation often requires placing a chest drain tube, unilateral or bilateral, in the pleura, pericardial cavity, or mediastinum (Khalkhali et al. 2014) to maintain hemodynamics and cardiovascular function (Zisis et al. 2015; Al-Otaibi et al. 2013). However, removing the tube causes pain (Kang et al. 2014).

Research in the past decade has shown that removing the chest drain tube produces significant pain (although short term) and has been known as a painful procedure throughout the world (Hood et al. 2014). In other words, it has been described as one of the most unpleasant memories experienced by patients during the postoperative period (Gorji et al. 2014). Pain is among the most common complications occurring after operation (Miller et al. 2010) and is significantly intense in the elderly patients (Kaye et al. 2010). Meanwhile, with increase in age, pain tolerance decreases, which leads to interference with daily life and dependence on spouses or significant others (Mirzamani et al. 2008). All these matters prove the importance of pain and its control and management (Sahbaei et al. 2014).

Considering that nurses are in direct and continuous contact with patients, they should assume an important role in assisting the patients to accept the drug-free treatments such as complementary and alternative medicine.
With regard to this responsibility, they (nurses) must first acquire adequate and complete information regarding the effectiveness of such therapy and its alternative, so that they would be able to teach it (Brolinson et al. 2001).

There are pharmacological and nonpharmacological methods to control pain. The pharmacologic approaches at the time of drain tube removal include using painkillers and sedatives, muscle relaxants, and non-steroidal anti-inflammatory drugs (EL-Gendy 2015).

Several decades ago, researchers developed pharmaceutical protocols for reducing pain arising from chest tube removal (Hood et al. 2014). They introduced it as the preferred method of treatment (Zisis et al. 2015). Nevertheless, studies have showed that the current protocols lack useful effect on managing pain, are undesirable, and should be revised (Hood et al. 2014). Meanwhile, pharmaceutical methods are expensive and have adverse effects on the cardiovascular system (Gutgsell et al. 2013).

Drug treatment in the elderly, as part of the physiological process of aging, causes degeneration of the autonomic nervous system and gradual decline in the ability to regulate cardiovascular responses (Wang et al. 2013). Pharmacokinetic changes put these patients at high risks (Bickley \& Szilagyi 2013), which is accompanied with side effects such as drowsiness, gastrointestinal bleeding, and addiction (Yeh et al. 2014; Yeh et al. 2013). Furthermore, using medications, especially in the elderly, is accompanied with complications because of their physical changes due to aging. Therefore, with restiction of using pharmaceuticals in recent years, drug-free treatments and complementary medicine are often preferred (Moemenabadi 2010).

Current studies have shown that the use of nonpharmacologic interventions have been very effective for prevention of pain (Al-Otaibi et al. 2013; Khalkhali et al. 2014; Hood et al. 2014). Nonpharmacologic methods to control pain are simpler and less expensive (Allred et al. 2010). A variety of nonpharmacologic interventions such as percutaneous electrical nerve stimulation (Johnson et al. 2015), acupuncture (Geib et al. 2015), acupressure (Chen et al. 2015), hypnosis therapy (Cordia et al. 2015), behavior therapy (Karlin et al. 2015), and massage therapy (Dreyer et al. 2015) have been emphasized. The results of a systematic review showed that acupressure was an effective and noninvasive method for relieving various types of pain and could be used as a training program in nursing curriculum (Chen \& Wang 2014). Among other nonpharmacological methods of pain relief is cryotherapy, which was first described by Hippocrates 
(Osunde et al. 2011). Also, there are behavioral-psychology methods such as deflection of the senses and music (Tuckettet al. 2015). Acupressure is one of the therapeutic relief approaches with many advantages that can be used by physicians, nurses, and even patients themselves and has had positive results in nursing interventions and patients' care (Rowbotham 2005).

Acupressure as a branch of acupuncture has been much discussed about (Mehdizadeh 2002; Aghamiri et al. 2005). It is noninvasive, has low cost, needs no device for its administration, is accessible compared to other medical methods, and eventually is easy to learn and can be carried out by the patients themselves or by their caregivers. As a result, it reduces the referrals to medical centers (Sadri et al. 2006). This alternative medicine was invented in China and focused on preserving the function of vital organs through circulation of blood and energy in the body (Chen \& Wang 2014). It sets in motion the energy flow, Chi, along 12 channels of energy in the body (Hamidzadeh et al. 2012). Any blockage along these channels leads to extremes of energy in the body and development of illness.

Hegu point, LI4, is one of the important pressure points related to the large intestine (Kaviani et al. 2012). It is located at the back of the hand between the first and second metacarpal bones, more precisely on the radial side of the second metacarpal bone. At this point, the energy current is close to the skin surface and can easily be stimulated by pressing (Hamidzadeh et al. 2012). Point ST36 or Zusanli point, is one of the most efficient acupoints associated with a wide range of effects such as analgesic and antispasmodic effects, and is the point where general tone is created (Stux et al. 2007). P6 or Nei guan point is located on the anterior surface of the forearm almost three fingers above the wrist between the flexor carpi radialis and palmaris longus tendons (Alessandrini et al. 2012). Several studies with different results have been conducted regarding the effect of acupressure on pain relief (Chen \& Wang 2014), labor pains (Akbarzadeh et al. 2015; Dabiri \& Shahi 2014), dysmenorrhea (EL-Gendy 2015; Wang et al. 2015), back pain (Akbarzadeh et al. 2015; Chen et al. 2015), fatigue (Eğlence et al. 2013), sleep (Simoncini et al. 2015), anxiety (McFadden et al. 2012), nasea and vomiting (Saberi et al. 2014), quitting smoking (Yeh et al. 2014), depression, constipation (Parker 2014), and pain of removing drain tube (Moemenabadi 2010). This type of medical approach is effective even in promoting spiritual health (Wein 2014).

Cryotherapy has been introduced as a noninvasive and nonpharmacological method. It is inexpensive and easily applicable to relieve the pain (Haynes 2015). Various methods of applying cryotherapy include using ice pack, ice massage, cold bath, and cooling gel packs. Cryotherapy, in its first minutes of application produces an uncomfortable sensation (Leventhal et al. 2010), but then the cold results in reducing the blood flow in the tissues and cell metabolism of the injured area (Modabber et al. 2013). It decreases vascular permeability (Dykstra et al. 2009), reduces inflammation and muscle pain (Salvo 2015) and eventually increases pain threshold (Hausswirth et al. 2011). In a study, it has been proven that, compared to other sedatives, cryotherapy has been effective for relieving postoperative pain (Zisis et al. 2015). In general, in a meta-analysis study, cryotherapy intervention has been introduced as an easy, affordable, and executable intervention (Wang et al. 2015). Another study also showed that the most effective method to apply cold for pain control is to use cryotherapy in localized form (Ertug \& Ulker 2012) and using cooling gel packs would lead to reduction in skin temperature, control of pain, and boosting pain threshold (Khalkhali et al. 2014)

Numerous studies with different results have been conducted on the effect of cryotherapy, for example, its effect on pain of removing the drain tube (Gorji et al. 2014; Payami et al. 2014), pain in breathing and deep coughing after undergoing open heart surgery (Khalkhali et al. 2014), pain of moving the drain tube where it is inserted, pain and anxiety when removing the drain tube (Zisis et al. 2015), pain and anxiety at the time of child delivery (Kaviani et al. 2012), and pain when taking ABG (Arterial Blood Gas) sampling.

In a study by Khorshid and Demir (2010) conducted on 90 patients undergoing open heart surgery, it was shown that cryotherapy was effective in reducing the intensity of pain during removal of the chest drain tube and has postponed the need for sedatives after removal of the drain tube (Demir \& Khorshid 2010). In another study conducted in 2013 by Al-Otaibi et al. on 40 patients hospitalized in the operation ward in a hospital in Saudi Arabia, the results showed that cryotherapy (cooling gel) had a significant effect on reducing pain and anxiety level when the chest drain tube was being removed (Zisis et al. 2015). Considering that pain is common and significantly high in the elderly (Eslami et al. 2016; Kaye et al. 2010), the findings of these two studies are encouraging. However, the samples selected for these two studies (Al-Otaibi et al. 2013; Demir \& Khorshid 2010) included patients over 18 years old and were not conducted solely on the elderly patients (Miller et al. 2010).

In general, nurses by implementing complementary medicine programs can play an important role in im- 
proving the quality of care and saving the treatment costs (Hsiung et al. 2015). Considering the palliative treatments for reducing pain of removing the drain tube and noting that different results have been reported on the effects of nonpharmacologic approaches in reducing pain arising from this procedure, the researchers decided to conduct a study in the ICU with the aim of comparing the effect of acupressure and cryotherapy on reducing the intensity of pain caused by removal of chest drain tube of the elderly who have undergone open heart surgery.

\section{Materials \& Methods}

This research was a quasi-experimental clinical trial in which 150 elderly patients who have undergone open heart surgery at Shahid Rajaei Hospital of Alborz Province were selected. Inclusion criteria were lacking any cognitive impairement or psychological disorders confirmed by using AMT (abbreviated mental test) tool and obtaining a score of at least 7 out of 10; having two chest drain tubes for at least 24 hours (one tube in the mediastinum and one in left pleura); not having received sedatives for 12 hours before the intervention; having normal range BMI (body mass index); lacking any scratch, scars, or disfigurement on the acupressure points of LI4, ST36, and SP6 in the acupressure group, not having received pain killers 4 hours before the intervention; lacking any cold sensitivity or allergy in cryotherapy group; not having serious illnesses such as diabetes, neuropathy, and so on; lacking drug addiction; and not using acupressure for any purpose.

The exclusion criteria included being under mechanical ventillation, suffering from severe vision or hearing disorders, having communication difficulties, sudden changes in the patient's status during the intervention, and pain in other organs and factors affecting the pain in the drain site (such as angina), and not feeling warmth, heaviness, swelling, or numbness during the application of acupressure on points ST36, LI4, and P6. However, no patient was excluded from this study.

Assigning samples to 3 groups (acupressure, cryotherapy, and control) were done through simple random sampling based on drawing lots containing the patients' names, in a way that the patient whose name was drawn first, was placed in the acupressure group, the second name in the cold therapy group, and the third one in the control group (50 patients in each group).

To collect data, a check list containing demographic data, abbreviated mental test (AMT), and visual analogue scale (VAS) of the pain was used. To validate demograph- ic questionnaire, AMT form, and pain VAS, the content validity method was used. In this regard, after careful review of the books, scientific journals, texts and essays on the subject of this research as well as using the opinions of student advisors, counselors, and statisticians, the researcher prepared the demographic data questionnaire and together with the AMT form and VAS, presented them to 10 faculty members of the School of Nursing and Midwifery of Iran University of Medical Sciences. After collecting and observing the opinions of the faculty members, required modifications were implemented. Then, research tools were presented to Ethics Committee of Iran University of Medical Sciences, and after their approval, those tools were employed in this study.

To determine the reliability of VAS, the Cronbach $\alpha$ coefficient was used to check its internal consistency. In this regard, 10 patients from the research population who had the profile of the elderly patients under the study filled out the questionnaires, and by using the results, the Cronbach $\alpha$ coefficient for VAS $(\mathrm{r}=0.85)$ was calculated. AMT is an appropriate test to screen the cognitive disorders in the elderly (Hodkinson 1972). This test has been widely used and its validity and reliability have been assessed in different countries. It has been assessed in Iran as regards its validation and showed a very high sensitivity and specificity in distinguishing patients with mild to moderate dementia from those with normal cognitive status. To assess the questionnaire's internal reliability, the Cronbach $\alpha$ coefficient was used which was equal to 0.76 . The tool sensitivity and specifity level based on DSM-IV (Diagnostic and Statistical Manual of Mental Disorders, $4^{\text {th }}$ Edition) criteria were 64.9 and 0.64 , respectively (Bakhtiyari 2014).

After assigning the samples in one of the three study groups, written consent forms were signed by them, and by asking questions from the patients, the checklist related to demographic information and also AMT was filled out by the researcher. Also, the intensity of pain was assessed by VAS which was used for gathering information prior to the intervention. Performing acupressure and cryotherapy was conducted by the researcher to prevent the bias as much as possible. Prior to the intervention in the acupressure group, the patients were asked to take deep breath and then the pressure was exerted on points ST36, P6, LI4 for a duration of 1 minute (with a sequence of 4 seconds of pressure and 2 seconds of rest). Generally, pressure was applied on each point for 10 minutes on both sides, and acupressure intervention was applied for 30 minutes in total (Chen et al. 2015). The researcher had been previously trained by specialists 
in acupressure and acupuncture about these points and held a certificate for carrying out acupressure.

The Hegu point or LI4 is located on the back of the hand between first and second metacarpal bones and more precisely on the radial side of the second metacarpal bone. At this point, the energy flow is closer to the skin surface and can be easily stimulated by finger pressure (Hamidzadeh et al. 2012). By closing one's fingers, the Hegu point is located precisely on the lower section of the fold created between first and second metacarpal bones (Andrews \& Dempsey 2011).

Point ST36 (Zusanli) with the width of a knuckle is located on the lateral and lower side of the tibial tuberosity 3 cuns (cun=Chinese unit of length, 1 cun is equal to $3.33 \mathrm{~cm}$ ) below the knee joint. In other words, it is located below the knee on the anterior tibialis muscle along the stomach meridian (4-finger width down from the bottom of the knee cap along the outer boundary of the shin bone). It is one of the most efficient points associated with a wide range of effects such as analgesia, anti-spasm, relieving fatigue, and generating a general tone (Stux et al. 2007).

The point P6 (Neiguan) with the width of $5 \mathrm{~cm}$ is located on the anterior forearm (in the proximal area toward the median plane) 2 cuns above the wrist crease between the tendons of palmaris longus and flexor carpi radialis muscles along the pericardium meridian. These points are known as acupressure points for analgesic effect and reducing postoperative pain (Hsiung et al. 2015).

On each point, pressure was applied by thumb for 4 seconds followed by 2 seconds of rest, and in each minute the cycle was repeated 10 times. This operation was carried out on both sides every 5 minute for 30 minutes (Chen et al. 2015). The accuracy of the point was confirmed when the patient felt warmth, heaviness, swelling, or numbness at that point (Moemenabadi 2010; Chen et al. 2015; Dabiri \& Shahi 2014). If the participants express severe pain in the location where pressure was applied, it was halted temporarily and renewed after a few minutes. It is worthy to note that since the average estimated time for the cycle of energy flow (meridian) through the body is 24 minutes, choosing 30 minutes for acupressure intervention was in order to ensure that a full cycle of energy flow through the body has been performed (Dabiri \& Shahi 2014).

In several studies, duration of time needed for acupressure intervention has been considered 30 minutes (Chen et al. 2015; Chen \& Wang 2014; Dabiri \& Shahi
2014). Pressure on each point under consideration was performed simultaneously in a symmetric fashion 20 minutes before removing the drain tube until 10 minutes after extubation. Attempts were made by repeating the application of pressure and using digital scale, to create a certain amount of pressure at each time. In this regard, after the researcher learned the procedure, a pressure equal to $3-5 \mathrm{~kg}$ under the right thumb and simultaneously a pressure equal to $3-5 \mathrm{~kg}$ under the left thumb was measured. To ensure that there would be no difference between pressures applied by the right and left thumbs, this pressure was calculated by employing the formula $\mathrm{P}=\mathrm{F} / \mathrm{A}$ (Where $\mathrm{P}$ is the force applied perpendicular to the surface of an object per unit area over which that force is distributed and is expressed in terms of $\mathrm{mm} \mathrm{Hg}, \mathrm{F}$ is the force in terms of $\mathrm{kg}$, and $\mathrm{A}$ is the area of the finger in $\mathrm{m}^{2}$ ) (Kaviani et al. 2012). To ascrtain of the accurate location of pressure points and the method of applying pressure, the concurrent validity was used.

In this way, the location of the pressure points and the method of applying pressure were approved by the acupuncturist. To check the reliability of the method, the reliability equivalence was used. As such, both the acupuncturist and the researcher applied pressure on the pressure points on 10 patients and the feelings resulting from the correct pressure, including warmth, heaviness, swelling or numbness were assessed and showed a high correlation between 2 mediators. Besides, removal of the chest drains was performed by expert nurses of the ICU in the morning shifts. It was done at the end of inhalation when a pause was made, and measurement of the drain tubes was based on calibrated lines on the tube. Moreover, measuring the distance between the location of the drain tube to the operation suture was carried out by the researcher using a calibrated ruler.

In the cryotherapy group, a cooling gel was placed on the site 20 minutes before removing the chest drains. Studies have shown that it requires 20 minutes to get the physiological effects in the cryotherapy method, and after that time, the reduction in the skin temperature reaches a constant level (Payami et al. 2014; Kol et al. 2013). In this study, applying cryotherapy was done through using 3 packs of cooling gel $\left(0^{\circ} \mathrm{C}, 8 \times 10 \mathrm{~cm}\right)$ (Bespar Iran Javidan Gostar Co.) (Gorji et al. 2014). They were applied on the periphery of the drain tube. In the control group, only the postoperative routine cares were performed.

In all groups, measurement of the pain intensity was recorded by the researcher's assistant (who had previously received required training) using the VAS criteria, 20 minutes before, immediately after, and 15 minutes after 
the removal of the chest drain tube. Examining the intensity of pain 15 minutes after drain removal was documented on several studies (Al-Otaibi et al. 2013; Khalkha ali et al. 2014; Gorji et al. 2014; Payami et al. 2014). The pain intensity 20 minutes before the intervention was compared among three groups. In all groups, after the intervention, collection of data (filling out demographic questionnaire and VAS questionnaire) was carried out. Patients were asked to reflect the intensity of pain that they experience on the VAS.

They were assured that acupressure and cryotherapy had no known complications and in case of any complication, the physician would be alerted. Also, if they were disinterested to take part in the study, they could exit which would not have any effect on their routine care. Furthermore, the same researcher performed the acupressure on all study patients, to prevent bias to the feasible extent. However, it was not possible that the researcher and all the elderly study patients be of the same gender, so to observe legal and Islamic ethics, the researcher used latex glove for applying acupressure and cryotherapy on all patients whether male or female (Rajaei et al. 2015). The obtained data were analysed using SPSS version 20. We performed the Chi-squared test, 1-way analysis of variance (ANOVA), the Fisher's exact test, repeated measures, and the Independent t-test.

\section{Results}

Findings showed that all study groups did not have significant differences as regards to age (Table 1), gender, educational degree, occupation, beliefs, type of operation, time passed from operation to the removal of the chest drain, and length of the chest drain tube. Furthermore, based on statistical analysis, no significant differ-

Table 1. Demographic characteristics of the elderly patients in 3 study groups.

\begin{tabular}{|c|c|c|c|c|c|}
\hline \multirow[t]{2}{*}{ Group } & \multirow[b]{2}{*}{ Variable } & Acupressure & Cryotherapy & Control & \multirow{2}{*}{$\begin{array}{c}\text { Significance } \\
\text { level* }\end{array}$} \\
\hline & & No.(\%) & No.(\%) & No.(\%) & \\
\hline \multirow{5}{*}{ Age(year) } & $60-62$ & $35(70)$ & $27(54)$ & $25(50)$ & \multirow{5}{*}{$P=0.24$} \\
\hline & $63-65$ & $8(16)$ & $11(22)$ & $16(32)$ & \\
\hline & & & & & \\
\hline & $66-68$ & $3(6)$ & $8(16)$ & $7(14)$ & \\
\hline & $69-71$ & $4(8)$ & $4(8)$ & 2(4) & \\
\hline \multirow{3}{*}{ Gender } & Male & $21(42)$ & $23(46)$ & $20(40)$ & \multirow{3}{*}{$P=0.82$} \\
\hline & & & & & \\
\hline & Female & $29(58)$ & $27(54)$ & $30(60)$ & \\
\hline \multirow{3}{*}{ Education } & Under diploma & $29(58)$ & $31(62)$ & $35(70)$ & \multirow{3}{*}{$P=0.44$} \\
\hline & & & & & \\
\hline & Diploma & $21(42)$ & $19(38)$ & $15(30)$ & \\
\hline
\end{tabular}

*Chi-square test.

Client-Centered Nursing Care

Table 2. The intensity of chest pain in 3 study groups, 20 minutes before removal of the chest drain.

\begin{tabular}{cccc}
\hline & Acupressure & Cryotherapy & Control \\
\cline { 2 - 4 } Intensity of pain & No.(\%) & No.(\%) & No.(\%) \\
\hline Without pain(0) & $4(8)$ & $4(8)$ & $7(14)$ \\
Slight(1-3) & $29(58)$ & $28(56)$ & $26(52)$ \\
Moderate(4-6) & $17(34)$ & $18(36)$ & $17(34)$ \\
Total & $50(100)$ & $50(100)$ & $50(100)$ \\
Mean \pm SD & $2.84 \pm 1.96$ & $2.98 \pm 2.14$ & $2.98 \pm 2.15$ \\
\hline
\end{tabular}


Table 3 . The intensity of pain in 3 study groups immediately after the removal of the chest drain tube.

\begin{tabular}{|c|c|c|c|c|}
\hline Group & & \multirow{2}{*}{$\begin{array}{c}\text { Acupressure } \\
\text { No.(\%) }\end{array}$} & \multirow{2}{*}{$\begin{array}{c}\text { Cryotherapy } \\
\text { No.(\%) }\end{array}$} & \multirow{2}{*}{$\begin{array}{l}\text { Control } \\
\text { No.(\%) }\end{array}$} \\
\hline & Intensity of pain & & & \\
\hline \multicolumn{2}{|r|}{ Slight(1-3) } & $13(26)$ & $6(12)$ & $0(0)$ \\
\hline \multicolumn{2}{|r|}{ Moderate(4-6) } & $28(56)$ & $21(45)$ & 2(4) \\
\hline \multicolumn{2}{|r|}{ Severe(7-9) } & $9(18)$ & $23(46)$ & $16(32)$ \\
\hline \multicolumn{2}{|r|}{ The most intense pain imaginable(10) } & $0(0)$ & $0(0)$ & $32(64)$ \\
\hline \multicolumn{2}{|r|}{ Total } & $50(100)$ & $50(100)$ & $50(100)$ \\
\hline \multicolumn{2}{|r|}{ Mean \pm SD } & $4.48 \pm 2.01$ & $5.86 \pm 2.20$ & $8.96 \pm 1.59$ \\
\hline & Results of analysis of variance & & $F=69.04$ & \\
\hline
\end{tabular}

Table 4 . The intensity of pain in 3 study groups, 15 minutes after removal of the chest drain tube.

\begin{tabular}{cccc}
\hline & Acupressure & Cryotherapy & Control \\
\cline { 2 - 4 } Intensity of pain & No.(\%) & No.(\%) & No.(\%) \\
\hline Without pain(0) & $8(16)$ & $2(4)$ & $0(0)$ \\
Slight(1-3) & $36(72)$ & $9(18)$ & $5(10)$ \\
Moderate(4-6) & $4(8)$ & $20(40)$ & 3(14) \\
Severe(7-9) & $2(4)$ & $19(38)$ & $50(100)$ \\
Total & $50(100)$ & $50(100)$ & $6.98 \pm 2.28$ \\
Mean \pm SD & $2.28 \pm 1.71$ & $4.80 \pm 2.20$ & P-value<0.001 \\
\hline
\end{tabular}

ence existed among three study groups regarding the intensity of pain before the intervention $(\mathrm{P}=0.93)$.

However, regarding the evaluation of the three groups in terms of the pain intensity immediately after and 15 minutes after removal of the chest drain tube, the results of Scheffe test showed significant differences among three groups (Tables 2 and 3). In the acupressure and cryotherapy groups, the intensity of pain was less than that in the control group. Also, the intensity of pain in the acupressure group was less than that in the cryotherapy group and the difference was significant $(\mathrm{P}<0.001)$. None of the elderly patients in all study groups immediately after the removal of the chest drain tube were without pain. However, 15 minutes after the removal of the chest drain, none of the elderly study patients in all groups experienced the most intense pain imaginable (Tables 3 and 4).
Based on the study results, majority of the patients $(56 \%)$ in the acupressure group felt moderate pain while many patients $(46 \%)$ in the cryotherapy group felt intense pain, and most patients (64\%) in the control group felt most intense pain imaginable (Table 3). In the acupressure and cryotherapy group, none of the elderly study patients experienced the most intense pain imaginable. These findings showed that the level of moderate pain in the acupressure group was more than that in the cold therapy group and the control group (56\% versus $42 \%$ and $4 \%$, respectively). However, the level of intense pain in the acupressure group was significantly lower than that in the cold therapy group and the control group (18\% versus $46 \%$ and $32 \%$, respectively). Table 3 compares the intensity of pain in all study groups. The Scheffe test shows significant differences before, immediately after, and 15 minutes after the removal of the chest drain tube (Figure 1). 


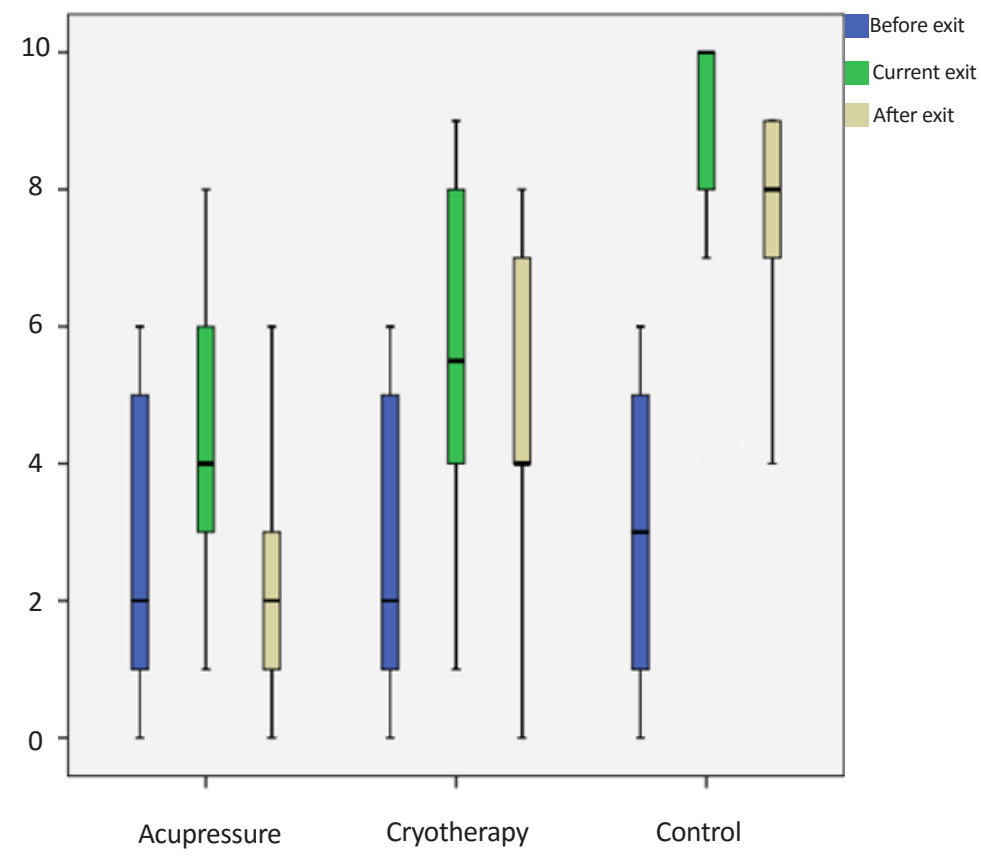

Client-Centered Nursing Care

Figure 1. Intensity of pain in 3 study groups, 20 minutes before, immediately after, and 15 minutes after removal of the chest drain tube.

\section{Discussion}

The present study has been conducted with the general aim of comparing the effectiveness of acupressure and cryotherapy on the pain resulting from removal of the chest drain tube of the elderly patients who have undergone open heart surgery. Control of pain in patients after heart surgery is one of the common problems in ICUs (Barr et al. 2013). Failure to treat the pain would deeply affect the quality of life and can have physical, psychological, social, and economic consequences. If acute pain is not properly managed, it can affect immune and nervous systems, and may progress and become chronic. Untreated pain can increase the risk of developing atelectasis, respiratory infections, myocardial ischemia, stroke, heart failure, and thromboembolic diseases in the patient (King \& Fraser 2013).

Apparently, use of a non-drug alternative (such as acupressure and cold therapy) with fewer side effects has always been appropriate in preventing postoerative pain. In this study, there were no significant difference in personal characteristics and type of illness, as well as in the intensity of pain before the removal of the drain tube among three study groups (Table 2). However, the Scheffe test results showed that there were significant differences among three groups (acupressure, cryotherapy, control) with regard to the intensity of pain immediately after, and 15 minutes after removal of the chest drain tube.

In the acupressure and cryotherapy groups, the intensity of pain was lower than the control group. Also, the intensity of pain in the acupressure group was lower than the cryotherapy group and this difference was significant $(\mathrm{P}<0.001)$ (Tables 3 and 4). Acupressure and cryotherapy were reported to be effective in other situations, too. Based on the intensity of pain in three groups, severe pain or the most intense pain imaginable was not reported in the groups before drain tube extubation (Table 2). However, immediately after removal of the tube, intense levels of pain with degrees of 7 to 9 and even 10 were reported in all groups (Table 3).

This finding is consistent with the results of another study reporting that the removal the chest drain is associated with severe pain (Hood et al. 2014). In other words, based on the study results, the most prevalent degree of pain $(56 \%)$ in the acupressure group was moderate pain, while the most prevalent type of pain in the cryotherapy group was intense pain (46\%), and the most prevalent type of pain in the control group was the most intense pain imaginable (64\%) (Table 3). Furthermore, in the acupressure and the cryotherapy groups, none of the study patients experienced the most intense pain imaginable. These findings indicate that the level of moderate 
pain in the acupressure group was more than that in the cryotherapy and control groups (56\% versus $42 \%$ and $4 \%$ respectively). However, the level of intense pain in the acupressure group was significantly lower than that in the cryotherapy and control groups (18\% versus $46 \%$ and $32 \%$, respectively) (Table 3 ).

Using the Scheffe test, a statistically significant difference was observed in the mean pain intensity of the study patients $(\mathrm{P}<0.001)$ immediately after removal of the drain. In other words, acupressure applied on points LI4, ST36, and P6 was effective in reducing pain after removal of the chest drain tube in the elderly patients, and with regard to the intensity of pain they showed significant difference compared to the cryotherapy and control groups. Considering the reports of significant pain (although of short duration) on removing the chest drain (based on the research done in the past decade) also its bad reputation as a painful procedure (Hood et al. 2014), no wonder that removal of the drain tube has been described as one of the most unpleasant memories experienced by patients during the postoperative period (Gorji et al. 2014) and in one study, patients have reported moderate to intense pain in this regard (Zisis et al. 2015). Therefore, our finding is considered as a positive outcome.

The highest level of pain, i.e., intense pain, was reported 15 minutes after removal of the chest drain tube by the control group (76\%), while the level of intense pain in the cryotherapy group was $38 \%$ and in the acupressure group was 4\%. Therefore, in the control group not only pain did not decrease, but in fact intense pain increased (76\%). However in the acupressure group, $16 \%$ of samples were without pain, while in the control group no patient was free of pain, and in the cryotherapy group only $2 \%$ of samples had no pain. Based on the Scheffe test, statistically significant differences were detected in the mean pain intensity in the study patients 15 minutes after removal of chest drain tube $(\mathrm{P}<0.001)$. In other words, in patients who received acupressure therapy at points ST36, LI4, and P6, the intensity of pain showed sigfnificant reduction compared with the other 2 groups 15 minutes after removal of the chest drain tube. Therefore, acupressure was able to reduce pain more at this stage.

Moreover, the degree of pressure applied on acupressure points and proper timing have decisive roles in reducing pain. In this regard, a high-frequency current activates special cells and through a byway triggers the endorphins producing system. In most cases high frequency current should be used for longer time because the duration of its effect is very short. On the other hand, low-frequency cur- rents has low analgesic effect at the beginning, but their effects last longer, diffuse and relieve pain more, and often associate with drowsiness. The analgesic effect of lowfrequency current rises during consecutive sessions and this phenomenon is due to enduring effect of endorphins triggered by low-frequency stimulation. In the long term, treatment with low-frequency current increases therapeutic effect (Jun et al. 2007).

Therefore, considering the study findings, applying acupressure on points SP6, LI4, and ST36 for 30 minutes can reduce pain immediately after removal of the chest drain tube and this phenomenon probably results from increase in the production of endorphins in the body. Likewise, in another study it has been concluded that the effect of interventions such as acupressure may include processes like stimulation of A delta fibers in the skin that reach the grey matter of the brain, inhibit pain stimulation and eventually reduce pain perception; activation of the enkephalins of brain neurons and inhibiting the transmission of pain to the brain; releasing endorphins and meta-enkephalins in the brain; activating pain relief system in midbrain; and affecting the central pain network in the hypothalamus and the limbic system (Ernst 2006).

Although our present study purpose was not to describe the neurophysiology of acupressure, but to examine the effect of acupressure and cold therapy in relieving or reducing pain caused by removal of chest drain tube in the elderly patients with open heart surgery, a constant presence at the patient's bedside to perform 30 minutes of acupressure has led to witnessing the positive results in the acupressure group. This factor can reduce anxiety and increase calmness in elderly patients. Moreover, our study results were consistent with the findings of Momenabadi (2010) study. Their study on 100 patients showed that the patients in the intervention group had significant reduction in the intensity of pain 15 minutes after application of acupressure as compared with the control group (Moemenabadi 2010).

Dabiri and Shahi reported that no significant differences existed among the 3 groups of acupressure (at point LI4), touch therapy, and the control group with regard to the duration and the intensity of labor pain, 30 minutes, 1 hour after the intervention, and every hour later until the end of the first phase of labor. However, in the acupressure group compared with the other two groups, there was significant reduction in the intensity of pain after the intervention. This means that acupressure was more effective in reducing pain compared with the other two groups (Dabiri \& Shahi 2014). This result is consistent with the our study 
results. In this study, the LI4 acuapoint was used for 30 minutes, which was one cycle of intervention.

Furthermore, results of Karimipour et al. (2011) study showed that there were no significant differences between the mean intensity of pain of the two study groups. Their results were not consistent with our study results. This inconsistency may be due to the type of pain (pain resulting from rheumatoid arthritis) and duration of performing acupressure intervention which was shorter than that in our study.

Another non-pharmacological procedure effective in control of pain is cryotherapy. Historically, therapeutic use of local or systemic cryotherapy was first described by Hippocrates (Osunde et al. 2011). After tissue damage, vasoactive agents such as histamine are released. These chemicals cause inflammation and leakage of fluid from blood vessels. Cryotherapy reduces inflammation through contraction of blood vessels and decreasing vasoactive agents of the damaged tissue. It allivates pain by boosting the pain threshold through slowing down the conduction velocity of neural pathways. In muscle tissues, cryotherapy eliminates fatigue by reducing muscle spasm through reduction in the activity of muscle spindle and peripheral nerves. Muscle spasms decrease by cold through a mechanism that is not well understood. Prolonged cold application on muscle spindle seems to be one of the reasons for reduction of muscle spasm. Muscle stiffness and spasm increases as a result of increased tissue viscosity and reduced collagen stretching tissue. One should be careful that application of cryotherapy should not last longer than 15 minutes. Lengthy and excessive cryotherapy will lead to compensatory vasodilation rather than vasoconstriction and eventually increase in edema and tissue damage (Jansen 2008).

Cold stimuli can increase delta fibers in the skin blood flow, alleviates headache (Weintraub et al. 2008), and calms the nerves (McCabe 2001). Cryotherapy results in reducing discomfort and pain in the damaged tissue, suppresses edema, and increases the recovery of the affected area (Andersen et al. 2015). Cold reduces inflammation and muscle pain (Salvo 2015), as well as activity of nociceptive receptors resulting in increased vasoconstriction (Scott \& Fishman 2012) and thus restriction of bleeding in the damaged area. It is also effective in reducing hyperthermia (Andersen et al. 2015). The aim of cryotherapy is to reduce pain, inflammation, and muscle spasm (Foreman et al. 2010; Jansen 2008). Al-Otaibi et al. (2013) conducted a study on 40 patients hospitalized in the surgeries special ward. They demonstrated that cryotherapy had a significant effect on reducing pain and anxiety at the time the removing chest drain tube (Zisis et al. 2015). Results of our study were consistent with this study. However, the duration of intervention in that study was shorter than that in our study.

Results of our study was consistent with results of the studies done by Demir and Khorshid (2010), Mazloum et al. (2012), and Kol et al. (2013). The reason for this consistency is application of cryotherapy for 20 minutes, because for obtaining the desired physiological effect of cryotherapy, it should be applied at least 20 minutes. Although in the study by Mazloum et al. (2012), ice pack and not cooling gel was used.

Our study results were similar to the findings of study conducted in 2014 by Dehghan and Farahbod. Their study results showed that the intensity of pain decreased in both groups of heat therapy and cold therapy. However, in the heat therapy group this reduction was more (Dehghan \& Farahbod 2014). Nevertheless, the study population and the type of pain in the two studies were different. Also, in their study, instead of cooling gel, ice pack was used.

The results of our study are in line with the study results of Haynes in 2015. His study showed that in two groups of cryotherapy, applying ice prior to ABG test had led to more pain relieve compared with two control groups. However, the difference between two groups of cryotherapy and two control groups was not significant. This study recommended application of cryotherapy as a drug-free, simple, and effective procedure without side effects (Haynes 2015). The reason for this consistency can be related to 20 minutes application of cryotherapy, because for obtaining the desirable physiological effects of cryotherapy, at least 20 minutes of its application is necessary. However, in that study, ice pack was used (not cooling gel). Moreover, the study population and type of pain were different in two studies.

Results of a study by Canbulat et al. (2015) showed that application of cryotherapy accompanied with vibration stimulus was effective in reducing pain and anxiety arising during venipuncture (Mirzamani et al. 2008). Results of our study was consistent with this study, too. However, the study population were different in two studies. The results of the present study was not consistent with the study by Witting-Wells et al. (2015). They conducted an RCT (Randomized Controlled Trial) study on patients undergoing knee joint replacement. After carrying out cryotherapy intervention, they concluded that no statistically significant difference was seen between two groups of cryotherapy and control (Wittig-Wells et al. 2015). Reason for inconsistency may be due to different study populations and the type of pain in two study groups. 
In addition, ice pack was used in that study. In a study by Khalkhali et al. (2014), the results indicated that the intensity of pain in the cryotherapy group was less than that in the control group and the difference was significant (Khalkhali et al. 2014). Results of our study are consistent with the findings of said study as regards the reduction in the intensity of pain. Such consistency is probably due to use of cooling gel by both studies to apply cryotherapy.

Results of a study by Heidari Gorji et al. (2013) conducted on 80 patients undergoing CABG at Mazanderan Province Heart Center Hospital, showed that there was no significant statistical difference among three groups as regards the intensity of pain after removal of the chest drain tube. However, the intensity of pain in the relaxation ice pack groups was lower than that in the control group (Gorji et al. 2014). Their results were not similar to our study results. However, in their study, the duration of cryotherapy was 10 minutes which was shorter than that duration in our study and they used ice pack instead of cooling gel.

With regard to studying the pain intensity immediately after and 15 minutes after removal of chest drain tube in three study groups (Tables 3 and 4), the results of Scheffe test showed that there were significant differences $(\mathrm{P}<0.001)$ among three groups, i.e. the mean score for the pain intensity in the control group was higher than in other two groups. Also, there was significant statistical difference between acupressure and cryotherapy groups, and the mean score of the pain intensity was higher in the cryotherapy group. In other words, application of acupressure will reduce pain immediately after and 15 minutes after the removal of the chest drain tube.

Thus, considering the mean scores in the two stages (immediately after, and 15 minutes after the removal of the drain tube), the intensity of pain decreased in groups receiving acupressure and cryotherapy. The mean intensity of pain in the acupressure group was 2.1 before the intervention and reached 4.2 immediately after, and then 2.1 fifteen minutes after removal of the drain tube. While before the intervention, the mean scores of pain intensity in the cryotherapy group and the control groups were 2.2 and 2.2, respectively. Then, they reached 5.2 and 8.1 , respectively immediately after, and 4.2 and 6.2, respectively 15 minutes after the drain tube removal. In general, the mean intensity of pain in the cryotherapy group decreased parallel to the pain intensity in acupressure group, thus the effectiveness of complementary medicine procedures such as acupressure and cryotherapy to reduce the intensity of pain should be acknowledged. However, the process of pain intensity reduction in acupressure group, considering its status before the intervention and at different intervals, had significantly been better and more obvious than the process of pain reduction in the cryotherapy group.

Results of our study did not conform with Haji Amini et al. (2012) study results (Hajiamini et al. 2012) and research by Kaviani et al. (2012). The reason for the inconsistency may be due to the different study populations, type of pain, and the manner by which cryotherapy was applied (ice massage instead of cooling gel). Also, in the mentioned studies only the LI4 point was used, and in Haji Amini study, duration of intervention was 20 minutes which with regard to the number of acupressure points and duration of the intervention were shorter than that in our study.

In another study by Ghaemmaghami et al. (2012), they showed that the intensity of pain decreased in both intervention groups. However, significant decrease was reported only in the cupping therapy intervention group (Ghaemmaghami et al. 2014). The results of that study was inconsistent with our study findings. The reason for inconsistency may be due to the different complications studied, difference in the selected acuapoints, duration of intervention, and the type of intervention procedure. However, the results of our study was in conformity with Sorour et al. (2014) study (Amany et al. 2014). That study showed that after the intervention, the acupressure group expressed significant decrease in pain compared with the other two groups (isometrics and control). However, the scores with regard to stiffness and physical disorder in the isometric group were significantly lower compared with the other two groups (acupressure and control). In general, there were no significant differences between the scores in the two groups. Although the study population and type of pain were different in two studies, it should be noted that duration of intervention in the above study was shorter than that in our study.

Based on the analysis of these findings, in the acupressure group, suppression of pain immediately after, and 15 minutes after removal of the chest drain tube can be due to effectiveness of acupressure applied on points ST36, LI4, and P6. Furthermore, results indicate that the intensity and duration of intervention (30 minutes) will suppress pain in the study patients and due to continuous stimuli of acupressure points, vital energy flow is augmented in the meridians and the therapeutic effects become effective. Although in analyzing this issue, limits of the study should be considered, too.

The novelty of these two procedures and lack of familiarity of the patients with them can be the source of some concerns, probably bewilderment, or even anxiety in the 
elderly patients, and affect the results. In this study, for preventing such concerns, the researcher and the head nurse (or the nurse worked in that ward) explained the safety and method of application of the procedures to the patients, so that these concerns be controlled to some extent.

Another limitation of this study is the different pain thresholds in various individuals. Such differences can be due to individual characteristics, cultural and social factors, as well as economic status and even the gender. Therefore, the intensity of pain may be expressed more than its real values. This is a kind of limitation that to some extent its management is beyond the researcher's control. Although by including the control group in our study, this limitation is somewhat resolved. Another limitation of this study relates to the skill and professional experience of the person in charge of removing the drain tube. It is one of the important factors involved in the intensity of pain during removing the chest drain. In our study, the patients were divided among four trained nurses of the medical ward who had sufficient proficiency and experience in this regard and therefore to a great extent, the said limitation was under control.

Because, this study was limited to patients who had undergone open heart surgery, its results cannot be generalized to all patients with had drain tubes (for reasons other than heart surgery). Also, the sampling method was non-random and can limit the external validity of this study. However, all operations were performed by one surgeon so that the difference in handling the tissues was minimized. Thus, we can assume that the differences in pain relief is only confined to the effectiveness of relief measures. Despite the mentioned limitations, this study has enriched the current literature regarding description and critical analysis of non-pharmacological interventions and potentially is effective for the elderly patients.

Acupressure points LI4 and P6, which have been used in this study are conveniently accessible. Because of ethical and cultural issues in our country, many women believe and insist on protecting their privacy and cover, so application of acupressure on the mentioned points had no contradiction with patients' cultural beliefs. Accordingly, these acupressure points are recommended due to their ease of access. The other point, i.e. ST36 requires a private environment so that it would lead to further cooperation of patients. In this research by using partitions this privacy was provided. Another strong point of our study was the equal time used for applying acupressure for each participant. Also, comparing the intensity of pain in two intervention groups (acupressure, cryotherapy) with a control group to measure the effect of control can be considered among the strong points of this study. In order to prevent bias, application of pressure was performed by one person.

Findings of this study can be important because of the attention paid to non-pharmacological measures in reducing pain due to removal of chest drain tube. It is recommended that application of acupressure be taken into account in clinical practice. In this regard, nurses should be trained through in-service training courses that can be included in their curriculum. It is also recommended that nurses during the patients' cares, allocate some time for applying this procedure or even teach it to the patients. Directors of medical centers can provide training and put this simple and low-cost care in care plannings and nurses' duties. This technique needs minimum facilities and without imposing additional costs on patients and treatment centers, reduce the intensity of pain experienced in these patients, and increase the quality of care considering the application of acupressure in a wide range of complications. Nurses, also, can teach acupressure and cryotherapy procedure to the patients' caregivers or their family to perform those tasks when it needed.

Application of acupressure and cryotherapy can reduce the consumption of sedatives and painkillers and consequently side effects of these drugs. It saves high costs of medication and medical advice for relief and control of pain. This is possible when suitable planning is carried out in this regard. Because the present study was conducted on elderly patients undergoing open heart surgery, we suggest that in future studies, the effect of acupressure be examined on reducing pain caused by other illnesses or operations on elderly patients.

Results of this study showed that the intensity of pain during removal of chest drain tube in the elderly patients undergoing open heart surgery decreased in both intervention groups (acupressure and cryotherapy). However, in the acupressure group, significant reduction of pain was reported. Acupressure applied on points ST36, LI4, and P6 seems to be an effective method for reducing pain caused by removal of chest drain tube in the elderly patients and is recommended as a safe, nonpharmacological, and inexpensive procedure to control pain.

Based on the review of the articles related to the present study, the research findings supported the application of traditional medicine for treatment of illnesses and so far no severe complications have been reported. Points LI4 and P6 used in this study were easily accessible. In our country, women believe and stress on protecting their privacy and cover; however, administration of 
cryotherapy and acupressure on those points was not in contradiction with their cultural beliefs. Therefore, using those points is recommended because of their easy access. The other point, ST36, needs a private place to entice patients' cooperation.

\section{Acknowledgments}

This study was financially supported by Vice Chancellor of Research and Technology of Iran University of Medical Sciences. Authors of this article express their gratitude to the director of Alborz Province Shahid Rajaei Hospital, also its nurses, and all patients who participated in this study.

\section{Conflict of Interest}

The authors declared no conflict of interests

\section{References}

Aghamiri, ZB, Vijeh, M, Latif, \& Nejad B 2005, '[Examining the effect of acupressure on the intensity of pain associated with primary dysmenorrhea (Persian)]', Hayat, vol. 11, no. 3-4, pp. 19-28.

Akbarzadeh, M, Masoudi, Z, Hadianfard, MJ, Kasraeian, M \& Zare, N 2015, 'Comparison of the effects of maternal supportive care and acupressure (BL32 acupoint) on pregnant women's pain intensity and delivery outcome', Women's Health Bulletin, 2014, p. 129208. doi: 10.1155/2014/129208

Alessandrini, M, Napolitano, B, Micarelli, A, de Padova, A \& Bruno, E 2012, 'P6 acupressure effectiveness on acute vertiginous patients: a double blind randomized study', Journal Alternative Complementary Medicine, vol. 18, no. 12, pp. 1121-26. doi:10.1089/acm.2011.0384

Allred, KD, Byers, JF \& Sole, ML 2010, 'The effect of music on postoperative pain and anxiety', Pain Management Nursing, vol. 11, no. 1, pp. 15-25. doi: 10.1016/j.pmn.2008.12.002

Al-Otaibi, RA, Mokabel, FM \& Al-Ghuneimy, Y 2013, 'The effect of cold application on pain and anxiety during chest tube removal', Journal of American Science, vol. 9, no. 7, pp. 13-23.

Amany, S, Amany, A \& Eman AEA 2014, 'Effectiveness of acupressure versus isometric exercise on pain, stiffness, and physical function in knee osteoarthritis female patients', Journal of Advanced Research, vol. 5, no. 2, pp. 193-200. doi: 10.1016/j. jare.2013.02.003

Andersen, HH, Poulsen, JN, Uchida, Y, Nikbakht, A, ArendtNielsen, L \& Gazerani, P 2015, 'Cold and L-menthol-induced sensitization in healthy volunteers-a cold hypersensitivity analogue to the heat/capsaicin model', Pain, vol. 156, no. 5, pp. 880-89. doi: 10.1097/j.pain.0000000000000123

Andrews, S \& Dempsey, B 2011, Acupressure and reflexology for dummies, Wiley \& Sons, New Jersy.

Bakhtiyari, F 2014, '[Validation of the Persian version of the abbreviated mental test (AMT) in elderly residents of kahri- zakcharity foundation (Persian)]', Iranian Journal of Diabetes and Metabolism, vol. 13, no. 6, pp. 487-94.

Barr, J, Fraser, GL, Puntillo, K, Ely, EW, Gélinas, C \& Dasta, JF et al. 2013, 'Clinical practice guidelines for the management of pain, agitation, and delirium in adult patients in the intensive care unit', Critical Care Medicine, vol. 41, no. 1, pp. 263-306.

Bickley, L \& Szilagyi, PG 2013, Barbara Bates' pocket guide to physical examination and history taking [A Emami Shushtari, Persian trans.], 7th edn, Jame`e Negar Publications, Tehran.

Brolinson, PG, Price, J, Ditmyer, M \& Reis, D 2001, 'Nurses' perceptions of complementary and alternative medical therapies', Journal of Community Health, vol. 26, no. 3, pp. 175-89. doi: 10.1023/a:101036901215

Canbulat, N, Ayhan, F \& Inal, S 2015, 'Effectiveness of externa cold and vibration for procedural pain relief during peripheral intravenous cannulation in pediatric patients', Pain Managment Nursing, vol. 16, no. 1, pp. 33-39.

Chen, HM, Wang, HH, Chiu, MH \& Hu, HM 2015, 'Effects of acupressure on menstrual distress and low back pain in dysmenorrheic young adult women: an experimental study', Pain Management Nursing, vol. 16, no. 3, pp. 188-97. doi: 10.1016/j. pmn.2014.06.002

Chen, YW \& Wang, HH 2014, 'The effectiveness of acupressure on relieving pain: a systematic review', Pain Management Nursing, vol. 15, no. 2, pp. 539-50. doi: 10.1016/j.pmn.2012.12.005

Cordia, MJ, Hirsigerb, S, Mérillatb, S \& Rascha, B 2015, 'Improving sleep and cognition by hypnotic suggestion in the elderly', Neuropsychologia, vol. 69, pp. 176-182. doi: 10.1016/j. neuropsychologia

Dabiri, F \& Shahi, A 2014, 'The effect of Li4 acupressure on labor pain intensity and duration of labor: a randomized controlled trial', Oman Medical Journal, vol. 29, no. 6, pp. 425-59. doi: $10.5001 /$ omj.2014.113

Dehghan, M \& Farahbod, F 2014, 'The efficacy of thermotherapy and cryotherapy on pain relief in patients with acute low back pain, a clinical trial study', Journal of Clinical and Diagnostic Research, vol. 8, no. 9, pp. 1-4. doi:10.7860/JCDR/2014/7404.4818

Demir, Y \& Khorshid, L 2010, 'The effect of cold application in combination with standard analgesic administration on pain and anxiety during chest tube removal: a single-blinded, randomized, double-controlled study', Pain Management Nursing vol. 11, no. 3, pp. 186-96.

Dreyer, NE, Cutshall, SM, Huebner, M, Fossc, DM, Lovely, JK Bauer, BA et al. 2015, 'Effect of massage therapy on pain, anxiety, relaxation, and tension after colorectal surgery: a randomized study', Complementary Therapies in Clinical Practice, vol. 21, no. 3, pp. 154-59. doi: 10.1016/j.ctcp.2015.06.004

Dykstra, JH, Hill HM, Miller, MG, Cheatham, CC, Michael, TJ \& Baker, RJ 2009, 'Comparisons of cubed ice, crushed ice, and wetted ice on intramuscular and surface temperature changes', Journal of Athletic Training, vol. 44, no. 2, pp. 136-41. doi $10.4085 / 1062-6050-44.2 .136$

Eğlence, R, Karataş, N \& Taşci, S 2013, 'The effect of acupressure on the level of fatigue in hemodialysis patients', Alternative Therapies in Health and Medicine, vol. 19, no. 6, pp. 23-31. 
EL-Gendy, SR 2015, 'Impact of acupressure on dysmenorrheal pain among teen-aged girls students', Wulfenia Journal, vol. 22, no. 2, pp. 218-25.

Eliopoulos, Ch 2014, Gerontological nursing, Lippincott Williams \& Wilkins, Philadelphia.

Ernst, E 2006, 'Acupuncture-a critical analysis', Journal of International Medicine, vol. 259, no. 2, pp. 125-37. doi: 10.1111/j.13652796.2005.01584.x

Ertug, N \& Ulker, S 2012, 'The effect of cold application on pain due to chest tube removal', Journal of Clinical Nursing, vol. 21, no. 5-6, pp. 784-90. doi: 10.1111/j.1365-2702.2011.03955.x

Eslami, V, Zimmerman, M, Grewal, T, Katz, M \& Lipton, R 2016, 'Pain and sleep disturbance in the elderly: Evaluation the role of stress and depression (P7.310)', International Journal of Geriatrics Psychiatry, vol. 31, no. 5, pp. 450-7. doi: 10.1002/gps.4349

Foreman, MD, Milisen, K \& Fulmer, TT 2010, Critical care nursing of older adults; Best Practices, Amazon Publication, New York.

Geib, J, Rieger, MA, Joos, S, Eschweiler, GW, Dresler, T \& Metzger, FG 2015, 'Introduction of auricular acupuncture in elderly patients suffering from major depression: protocol of a mixed methods feasibility study', BioMed Research International. doi: 10.1155/2015/678410

Ghaemmaghami, M, Akbarzadeh, M, Yazdanpanahi, Z, Zare, N, Azizi, A \& Mohagheghzadeh, A 2014, '[Comparing the effect of dry cupping therapy and acupressure at bl23 point on the intensity of perineal pain after childbirth in primipara women (Persian)]', Qom University of Medical Sciences Journal, vol. 8, no. 6, pp. 26-31.

Gorji, HM, Nesami, BM, Ayyasi, M, Ghafari, R \& Yazdani, J 2014, 'Comparison of ice packs application and relaxation therapy in pain reduction during chest tube removal following cardiac surgery', North American Journal of Medical Sciences, vol. 6, no. 1, pp. 19-24. doi: 10.4103/1947-2714.125857

Gutgsell, KJ, Schluchter, M \& Margevicius, S 2013, 'Music therapy reduces pain in palliative care patients: a randomized controlled trial', Journal of Pain and Symptom Management, vol. 45, no. 5, pp. 822-31. doi: 10.1016/j.jpainsymman.2012.05.008

Hajiamini, Z, Nir Masoud, S, Ebadi, A, Afzali, M \& Matin, AA 2012, 'Comparing the effects of ice massage and acupressure on labor pain reduction', Complementary Therapies in Clinical Practice, vol. 18, no. 3, pp. 169-72. doi: 10.1016/j. ctcp.2012.05.003

Hamidzadeh, A, Shahpourian, F, Orak, R, Montazeri, AS \& Khosravi, A 2012, '[Effects of LI4 acupressure on labor pain in the first stage of labor (Persian)], Journal of Midwifery \& Women's Health, vol. 57, no. 2, pp. 133-38.

Hausswirth, Ch, Louis, J, Bieuzen, F, Pournot, H, Fournier, J, Filliard, JR et al. 2011, 'Effects of whole-body cryotherapy vs. far-infrared vs. passive modalities on recovery from exerciseinduced muscle damage in highly-trained runners', PLoS One, vol. 6, no. 12, p. e2774. doi: 10.1371/journal.pone.0027749

Haynes, JM 2015, 'Randomized controlled trial of cryoanalgesia (ice bag) to reduce pain associated with arterial puncture', Respiratory Care, vol. 60, no. 1, pp. 1-5. doi: 10.4187/respcare.03312

Hodkinson, HM 1972, 'Evaluation of a mental test score for assessment of mental impairment in the elderly', Age and Ageing, vol. 1, no. 4, pp. 233-8. doi: 10.1093/ageing/1.4.233
Hood, BS, Henderson, W \& Pasero, C 2014, 'Chest tube removal: an expanded role for the bedside nurse', Journal of Perianesthesia Nursing, vol. 29, no. 1, pp. 53-59. doi 10.1016/j. jopan.2013.11.001

Hosseinian, A, Kasayi, V, Mohammadzade, AR, Habibzadeh, S, Saghi, F \& Davari, M 2014, '[Evaluation of early complications Coronary Artery Bypass Grafting Surgery (CABGS) in the first month after operation in Imam Khomeini Hospital of Ardabil during 2013-2014 (Persian)]', Journal of Ardabil University of Medical Sciences, vol. 14, no. 1, pp. 18-27.

Hsiung, WT, Chang, YC, Yeh, ML \& Chang, YH 2015, 'Acupressure improves the postoperative comfort of gastric cancer patients: a randomised controlled trial', Complementary Therapies in Medicine, vol. 23, no. 3, pp. 339-46. doi: 10.1016/j. ctim.2015.03.010

Jansen, MP 2008, Managing pain in the older adult, Elsevier Publication, New York.

Johnson MI, Paley, CA, Howe, TE \& Sluka, KA 2015, 'Transcutaneous electrical nerve stimulation for acute pain', The Cochrane Database Systematic Reviews, vol. 15, no. 6, p. CD006142. doi: 10.1002/14651858.CD006142.pub3

Jun, EM, Chang, S, Kang, DH \& Kim, S 2007, 'Effects of acupressure on dysmenorrhea and skin temperature changes in college students: a non-randomized controlled trial', International Journal of Nursing Studies, vol. 44, no. 6, pp. 973-81. doi: 10.1016/j.ijnurstu.2006.03.021

Kang, H, Chung, YS, Choe, JW, Woo, YC, Kim, SW, Park, SJ et al. 2014, 'Application of lidocaine jelly on chest tubes to reduce pain caused by drainage catheter after coronary artery bypass surgery', Journal of Korean Medical Science, vol. 29, no. 10, pp. 1398-403. doi 10.3346/jkms.2014.29.10.1398

Karimipour, F, Fayyazi, S, Maula, K \& Latifi, M 2011, '[Effect of acupressure on pain intensity of patients with rheumatoid arthritis (Persian)], Jundishapur Scientific Medical Journal, vol. 11, no. 3, pp. 269-275.

Karlin, BE, Trockel, M, Spira, AP, Taylor, CB \& Manber, R 2015, 'National evaluation of the effectiveness of cognitive behavioral therapy for insomnia among older versus younger veterans', International Journal of Geriatric Psychiatry, vol. 30, no. 3, ,pp. 308-15. doi: 10.1002/gps.4143

Kaviani, M, Ashuri, M, Azima, S, Rajaeifard, A \& Hadianfard, MJ 2012, '[Comparing the effect of the two procedures-acupressure and ice massage at LI4 point on the intensity of pain, anxiety level and labor length (Persian)]', Journal of Shahid Sadoughi University of Medical Sciences, vol. 20, no. 2, pp. 220-28.

Kaye, AD, Baluch, A \& Scott, JT 2010, 'Pain management in the elderly population: a review', Ochsner Journal, vol. 10, no. 3, pp. 179-87.

Khalkhali, H, Tanha, ZE, Feizi, A \& Ardabili, SS 2014, 'Effect of applying cold gel pack on the pain associated with deep breathing and coughing after open heart surgery', Iranian Journal of Nursing and Midwifery Research, vol. 19, no. 6, pp. 545-49.

King, NB \& Fraser, V 2013, 'Untreated pain, narcotics regulation, and global health ideologies', PLoS Medicine, vol. 10, no. 4, p. e1001411. doi: 10.1371/journal.pmed.1001411

Kol, E, Erdogan, A, Karsli, B \& Erbil, N 2013, 'Evaluation of the outcomes of ice application for the control of pain associated 
with chest tube irritation', Pain Management Nursing, vol. 14, no. 1, pp. 29-35.

Leventhal, LC, Bianchi, RC \& de Oliveira, SM 2010, 'Clinical trial comparing three types of cryotherapy in nonpregnant women', Revista da Escola de Enfermagem da USP, vol. 44, no. 2, pp. 339-45. doi: 10.1590/s0080-62342010000200014

Mazloum, SR, Abbasi Teshnizi, M, Kianinejad, A \& Gandomkar, F 2012, 'Effect of applying ice bag on pain intensity associated with chest tube removal after cardiac surgery', Horizon Medical Science, vol. 18, no. 3, pp. 109-14.

McCabe, P 2001, Complementary therapies in nursing and midwife$r y$, Ausmed Pub, Melbourne.

McFadden, KL, Healy, KM, Hoversten, KP, Ito, TA \& Hernández, TD 2012, 'Efficacy of acupressure for non-pharmacological stress reduction in college students', Complementary Therapies Medicine, vol. 20, no. 4, pp. 175-82. doi: 10.1016/j. ctim.2011.12.003

Mehdizadeh, J 2002, [A guide to acupressure (Persian)], $1^{\text {st }}$ edn, Noore Danesh Publications, Tehran.

Miller, RD, Cucchiara, RF \& Miller, FD 2010, Miller's Anesthesia, $7^{\text {th }}$ edn, Churchill Li-vingston, Philadelphia.

Mirzamani, M, Holisaz, MT, Sadidi, A \& Safari B 2008, '[A Comparison of the effect of pain on various dimensions of the life of older people and people with chronic pain (Persian)]', Iranian Journal of Ageing, vol. 3, no. 1, pp. 48-57.

Modabber, A, Rana, M, Ghassemi, A, Gerressen, M, Gellrich, NC, Hölzle, F et al. 2013, 'Three-dimensional evaluation of postoperative swelling in treatment of zygomatic bone fractures using two different cooling therapy methods: a randomized, observer-blind, prospective study', Trials, vol. 1, no. 14, p. 238. doi: 10.1186/1745-6215-14-238

Moemenabadi, H 2010, [Examining the effect of acupressure on the intensity of pain and physiologic indicators resulting from removal of chest drainage tube at ICU of Shariati Hospital (Persian)], MA thesis, Iran University of Medical Sciences.

Osunde, OD, Adebola, RA \& Omeje, UK 2011, 'Management of inflammatory complications in third molar surgery: a review of the literature', African Health Sciences, vol. 11, no. 3, pp. 530-537.

Parker, J 2014, Acupressure and reflexology for dummies: mind and body relaxation, Wiley \& Sons Publications, New Jersey.

Payami, MB, Daryei, N, Mousavinasab, N \& Nourizade, E 2014, '[Effect of cold application in combination with Indomethacin suppository on chest tube removal pain in patients undergoing open heart surgery (Persian)]', Iranian Journal of Nursing $\mathcal{E}$ Midwifery Research, vol. 19, no. 1, pp. 77-81.

Rajaei, N, Choopani, N, Pishgoie, AH \& Sharififar, S 2015, '[Examining the effect of acupressure on anxiety in patients scheduled to receive coronary angiography (Persian)]', Journal of Military Care Sciences, vol. 2, no. 1, pp. 6-13.

Rouch, I, Achour Crawford, E, Roche, F, Castro Lionard, C, Laurent, B, Ntougou Assoumou, G et al. 2014, 'Seven-year predictors of self-rated health and life satisfaction in the elderly', Journal of Nutrition, Health \& Aging, vol. 18, no. 9, pp. 840-47. doi: 10.1007/s12603-014-0557-6

Rowbotham, D 2005, 'Recent advances in the non-pharmacological management of postoperative nausea and vomiting', Brit- ish Journal of Anesthesia, vol. 95, no. 1, pp. 77-81. doi: /10.1093/ bja/aei125

Saberi, F, Sadat, Z, Abedzadeh Kalahroudi, M \& Taebi, M 2014 'Effect of ginger on relieving nausea and vomiting in pregnancy: a randomized, placebo-controlled trial', Nursing $\mathcal{E}$ Midwifery Studies, vol. 3, no. 1, p. e11841. doi: $10.17795 / \mathrm{nm}$ sjournal11841

Sadri, B, Nematullahi, M, \& Shahrami, R 2006, '[The effect of acupressure on reducing nausea and vomiting after adenotonsillectomy (Persian)]', Journal of Iran University of Medical Sciences, vol. 13, no. 53, pp. 121-26.

Sahbaei, F, Abedini, Sh, Ghandehari, H \& Zare, M 2014, '[Examining the effect of foot reflexology massage on the pain of patients with scoliosis undergoing spinal surgery (Persian)]', Scientific Journal of Anesthesiology \& Pain, vol. 5, no. 2, pp. 63-71.

Salvo, SG 2015, Massage therapy: principles and practice, $5^{\text {th }}$ edn, Elsevier Publications, New York.

Scott, M \& Fishman, S 2012, Bonica's management of pain, Lippincott Williams \& Wilkins, Philadelphia.

Simoncini, M, Gatti, A, Quirico, PE, Balla, S, Capellero, B, Obialero, $R$ et al. 2015, 'Acupressure in insomnia and other sleep disorders in elderly institutionalized patients suffering from Alzheimer's disease', Aging Clinical \& Exprimental Research, vol. 27 , no. 1, pp. 37-42.

Stux, G, Berman, B \& Pomeranz, B 2007, Basics of acupuncture [B Naderi Nabi, A Sedighinejad, Persian trans.]', Teymurzadeh Publications, Tehran.

Tuckett, AG, Hodgkinson, B, Rouillon, L, Balil-Lozoya, T \& Parker, D 2015, 'What carers and family said about music therapy on behaviours of older people with dementia in residential aged care', International Journal of Older People Nursing, vol. 10, no. 2, pp. 146-57. doi: 10.1111/opn.12071

Wang, L, Gu, Z, Zhai, R, Zhao, S, Luo, L, Li, D et al. 2015, 'Efficacy of oral cryotherapy on oral mucositis prevention in patients with hematological malignancies undergoing hematopoietic stem cell transplantation: a meta-analysis of randomized controlled trials', PLoS One, vol. 10, no. 5, p. e0128763. doi: 10.1371/journal.pone. 0128763

Wein, J 2014, 'Self-healing-cervical spondylosis: acupressure to relieve cervical spondylosis', viewed 24 June 2015, https:/ / books.google.com/books?id=uwzvAwAAQBAJ

Weintraub, MI, Mamtani, R \& Micozzi, MS 2008, Complementary and Integrative Medicine in Pain Management, Elsevier Publication, New York.

Wittig-Wells, D, Johnson, I, Samms-Mc Pherson, J, Thankachan S, Titus, B, Jacob, A et al. 2015, 'Does the use of a brief cryotherapy intervention with analgesic administration improve pain management after total knee arthroplasty?', Orthopaedic Nursing, vol. 34, no. 3, pp. 148-53. doi: 10.1097/ nor.0000000000000143

Yeh, CH, Chien, LC, Balaban, D, Sponberg, R, Primavera, J, Morone, NE et al. 2013, 'A randomized clinical trial of auricular point acupressure for chronic low back pain: a feasibility study', Evidence-Based Complementary \& Alternative Medicine, pp. 1-13. doi: 10.1155/2013/196978

Yeh, $\mathrm{CH}$, Morone, NE, Chien, LC, Cao, Y, Lu, H, Shen, J et al. 2014, 'Auricular point acupressure to manage chronic low 
back pain in older adults: a randomized controlled pilot study', Evidence-Based Complementary \& Alternative Medicine, 2014, pp. 375173. doi: $10.1155 / 2014 / 375173$

Zisis, Ch, Tsirgogianni, K, Lazaridis, G, Lampaki, S, Baka, S, Mpoukovinas, I et al. 2015, 'Chest drainage systems in use', Annals of Translational Medicine, vol. 3, no. 3, p. 43. doi: 0.3978/j.issn.2305-5839.2015.02.09 